\title{
Influences of Long-Term, High-Dose Acetaminophen Administration on Liver Function Markers in Healthy Japanese Adults
}

\author{
Ildae SONG ${ }^{* 1, * 2}$, Rieko TANAKA ${ }^{* 1}$, Masako ASO ${ }^{* 3}$, Yasutoshi SAKAMOTO*1 ${ }^{* 1}$ Mika MAEDA ${ }^{* 2, * 3, * 4}$, \\ Michiru OCHIAI $^{* 2}$, Yoshiro SAITO ${ }^{* 5}$, Keiko MAEKAWA ${ }^{* 5, * 6}$ and Yuji KUMAGAI ${ }^{* 1, * 3}$
}

\begin{abstract}
Background: Acetaminophen is widely used as an analgesic and antipyretic; however, acetaminophen overdose is known to cause hepatic injury. However, minor and self-limiting alanine aminotransferase (ALT) elevation unrelated to hepatic injury is occasionally observed in individuals receiving high-dose acetaminophen. The aim of this study was to evaluate the changes in liver function markers induced by long-term, highdose acetaminophen administration.

Methods: Acetaminophen $(3000 \mathrm{mg} /$ day $)$ or placebo was repeatedly administered to 242 healthy Japanese adults for 28 days. Plasma samples collected on Day 1 were used to measure the pharmacokinetics of acetaminophen. Liver function was monitored in terms of aspartate aminotransferase (AST), ALT, alkaline phosphatase (ALP), total bilirubin (T-Bil), and high mobility group box 1 (HMGB-1) levels for 35 days, from the day of the first dose. Subjects were withdrawn from the study if their AST, ALT, or ALP levels exceeded twice the respective upper limit of normal $(2 \times \mathrm{ULN})$.

Results: From a total of 242 subjects, 202 and 40 subjects were assigned to the acetaminophen group and the placebo group, respectively. Twelve subjects in the acetaminophen group $(6.0 \%)$ were withdrawn owing to ALT elevation over $2 \times$ ULN; no subjects were withdrawn from the placebo group. During the study period, ALT was higher in the acetaminophen group than in the placebo group, and increased from Day 7 to 14 after the start of administration. However, no evidence of hepatic injury owing to acetaminophen was observed, and the ALT elevation was attenuated after Day 14. Moreover, no correlation was observed between maximum ALT and levels of HMGB-1, a novel biomarker candidate for hepatic injury, during the study period. These findings led us to conclude that the ALT elevation was not caused by hepatic injury.

Conclusion: ALT elevation $>2 \times$ ULN was observed in $6.0 \%$ of subjects in the acetaminophen group. However, no subjects developed hepatic injury, and ALT levels started to return to the normal values even during continued administration. The phenomenon of adaptation may be involved in these changes.
\end{abstract}

Key words : acetaminophen, DILI, liver function test, adaptation

Jpn J Clin Pharmacol Ther. 2017; 48 (5) : 153-159

\section{Introduction}

Acetaminophen is widely used as an analgesic and antipyretic; however, acetaminophen overdose $(\geq 150 \mathrm{mg} / \mathrm{kg}$ single dose in adults) is known to cause liver injury. ${ }^{1-3)}$ In 2011 , the approved maximum daily dose in Japan was increased from $1500 \mathrm{mg}$ to $4000 \mathrm{mg}$, which is the same as in other foreign countries; this led to an increased concern among Japanese officials about the induction of liver injury in patients receiving high-dose regimens of acetaminophen, as well as drug safety.

In liver function tests, elevated levels of alanine aminotransferase (ALT), aspartate aminotransferase (AST), alkaline phosphatase (ALP), total bilirubin (T-Bil), and other liver function markers are often used to diagnose hepatic disease. However, it is well known that the minor elevation of these indicators does not necessarily signify hepatic injury. Serum high mobility group box 1 (HMGB-1), in contrast, has gained attention as a novel biomarker for hepatic disease: it is reportedly increased in the early stages of hepatic disease, more responsive than ALT, and correlated with ALT at the most severe stages of disease. ${ }^{4}$

In recent years, cases of minor ALT elevation in patients without apparent liver injury have been reported, ${ }^{5)}$ but hepatic injury onset during a high-dose acetaminophen regimen has never been observed. In such cases, the elevation of liver function tests are known to be transient and self-limiting and return to normal level even under continuous administration of acetaminophen in almost all cases. ${ }^{6)}$

Therefore, we investigated changes in the pattern of liver function markers, such as ALT, and factors related to the change of ALT after a long-term high-dose acetaminophen administration investigated in Japanese healthy adults.

\section{Subjects and methods}

\section{Subjects}

Healthy Japanese adult volunteers who satisfied the following criteria, and did not meet the exclusion criteria below, were included in the study: age, $\geq 20$ and $<45$ years; weight, $\geq 50$ $\mathrm{kg}$ and $<100 \mathrm{~kg}$ (male) or $\geq 50 \mathrm{~kg}$ and $<80 \mathrm{~kg}$ (female) ; BMI,

\footnotetext{
${ }^{* 1}$ Kitasato University School of Medicine, Kitasato Clinical Research Center, Japan $\quad{ }^{* 2}$ Kitasato University Graduate School of Medical Sciences, Japan ${ }^{* 3}$ Kitasato University Hospital, Clinical Trial Center, Japan ${ }^{* 4}$ Kitasato University Hospital, Department of Pharmacy, Japan $\quad{ }^{* 5}$ Division of Medicinal Safety Science National Institute of Health Sciences, Japan $\quad{ }^{* 6}$ Doshisha Women's University, Faculty of Pharmaceutical Sciences, Japan

Address for correspondence : KUMAGAI Y. Kitasato University School of Medicine, Kitasato Clinical Research Center, 1-15-1 Kitasato, Minami-ku, Sagamihara, Kanagawa 252-0375, Japan E-mail : kuma-guy@za2.so-net.ne.jp

Manuscript received May 23, 2017; revised July 25, 2017; accepted August 1, 2017

ISSN 0388-1601 Copyright: (C2017 the Japanese Society of Clinical Pharmacology and Therapeutics(JSCPT)
} 




Figure 1 Subject flow diagram

$\geq 18 \mathrm{~kg} / \mathrm{m}^{2}$ and $<27 \mathrm{~kg} / \mathrm{m}^{2}$; AST and ALT levels: < upper limit of normal (ULN).

Subjects with any of the following indications were excluded: acetaminophen hypersensitivity (past); peptic ulcers, blood abnormalities, liver disease, renal disease, cardiac insufficiency, or aspirin-induced asthma (current or past) ; regular consumption of large quantities of alcohol; positive result for $\mathrm{HBs}$ antigen or HCV antibody test; or pregnancy, breastfeeding, or potential pregnancy.

This study was reviewed and approved by the Institutional Review Board of Kitasato University (Sagamihara) and conducted adults in conformance with the ethical principles of the Declaration of Helsinki and with the Ethical Guidelines for Medical Research Involving Human Subjects (December 22, 2014; henceforth referred to as Ethical Guidelines). Study volunteers received a full explanation, both oral and written, of all information specified in the Ethical Guidelines, including the study objectives and methods, expected clinical risks, and inconveniences. Volunteers who gave consent to participate in this study were registered as subjects. This study was registered with the University Hospital Medical Information Network (UMIN-CTR) (UMIN study ID: 000019607).

\section{Study design}

This was a randomized, single-blind, placebo-controlled, repeated-dose study. Subjects were assigned to either the acetaminophen group or the placebo group by using block randomization at a $5: 1$ ratio (acetaminophen : placebo).

\section{Drug regimen}

Subjects were administered acetaminophen or placebo for 28 consecutive days. On Day 1, a single dose of acetaminophen $(500 \mathrm{mg} \times 2$ tablets $)$ or placebo was taken orally under fasting conditions. From Day 2 to Day 28, the same dose was taken after meals three times daily. Subjects were admitted to the Kitasato
University Hospital Clinical Trial Center from Day 1 to Day 7 for observation. After discharge, subjects were continuously administered their respective drug until Day 28, and observed until Day 35. To ensure safety, subjects were withdrawn from the study if levels of ALT, AST, or ALP exceeded twice the respective upper limit of normal (ULN) during the study period (respective $\geq 2 \times$ ULN values of ALT $\geq 80$, AST $\geq 80$, ALP $\geq 718$ ).

\section{Evaluation items and hepatic injury criteria 4.1. Evaluation items}

The levels of ALT, AST, ALP, T-Bil, and HMGB-1 were evaluated as liver function markers. In all subjects, these liver function markers were measured on Days 1, 2, 4, 7, 14, 21, 28, and 35 after the start of administration of the study drug.

\subsection{Hepatic injury criteria}

The development of hepatic injury was determined by using Hy's law criteria, promulgated by the FDA as a prognostic indicator for the evaluation of the risk of serious drug-induced hepatic injury. ${ }^{7)}$

\section{Pharmacokinetics}

Blood was sampled into vacuum blood collection tubes (containing heparin) before administration of the study drug on Day 1, and at $0.5,1,2,4,6,8$ and $12 \mathrm{~h}$ after administration. Plasma was isolated by centrifugation $\left(3,000 \mathrm{rpm}, 4^{\circ} \mathrm{C}, 10 \mathrm{~min}\right)$, and cryopreserved below $-70^{\circ} \mathrm{C}$ until analysis. The plasma concentration of unchanged acetaminophen was measured by a highperformance liquid chromatography/tandem mass spectrometry (HPLC-MS/MS) method. The pharmacokinetic parameters of acetaminophen $\left(\mathrm{C}_{\max }\right.$, peak serum concentration; $\mathrm{AUC}_{0-12}$, area under the serum concentration-time curve from 0 to 12 hours after administration; and $\mathrm{CL} / \mathrm{F}$, total clearance) were computed using Phoenix WinNonlin v. 6.4, according to a noncompartmental analysis model. 
Table 1 Subject characteristics at baseline

\begin{tabular}{lccc}
\hline & $\begin{array}{c}\text { Acetaminophen } \\
\mathrm{N}=202\end{array}$ & $\begin{array}{c}\text { Placebo } \\
\mathrm{N}=40\end{array}$ & P value \\
\hline Male, N (\%) & $135(66.8)$ & $26(65.0)$ & 0.855 \\
Age, mean \pm SD, years & $31.9 \pm 8.1$ & $30.8 \pm 7.4$ & 0.420 \\
BMI, mean \pm SD & $22.0 \pm 2.1$ & $22.0 \pm 1.8$ & 0.900 \\
Self-reported ethanol use N $(\%)$ & $114(56.4)$ & $26(65.0)$ & 0.318 \\
Baseline ALT, mean \pm SD & $17.5 \pm 9.3$ & $14.9 \pm 7.3$ & 0.092 \\
Baseline AST, mean \pm SD & $17.6 \pm 4.7$ & $17.1 \pm 5.6$ & 0.500 \\
\hline
\end{tabular}

Categorical data are shown as percentage of subjects. Continuous data are shown as mean and standard deviation. $\mathrm{P}$ value were calculated by Fisher's exact test for categorical data or t-test for continuous data.

Table 2 Liver function markers of male and female at baseline

\begin{tabular}{lccc}
\hline & $\begin{array}{c}\text { Male } \\
\mathrm{N}=161\end{array}$ & $\begin{array}{c}\text { Female } \\
\mathrm{N}=81\end{array}$ & P value \\
\hline Baseline ALT, mean $\pm \mathrm{SD}$ & $19.8 \pm 9.5$ & $11.6 \pm 4.4$ & $<0.001$ \\
Baseline AST, mean $\pm \mathrm{SD}$ & $18.4 \pm 5.2$ & $15.7 \pm 3.5$ & $<0.001$ \\
\hline
\end{tabular}

$P$ value was calculated by t-test.

\section{Sample size}

A previous study reported a $1 \%$ incidence of ALT elevation $>3$ $\times$ ULN, for which a causal link with acetaminophen could not be excluded, after the administration of a high-dose acetaminophen regimen for 4-24 weeks. ${ }^{8)}$ We therefore selected a sample size of 200 for the acetaminophen group in order to obtain 3-5 subjects with a mild ALT elevation $(2-3 \times U L N)$. To observe a day-today variation of aminotransferase (ALT, AST), the placebo group (without the administration of acetaminophen) was used as a control in this study. We selected a sample size of 40 for the placebo group, which was a feasible number of subjects. Accordingly, the overall sample size was 242 subjects (acetaminophen group, 202; placebo group, 40).

\section{Statistical analysis}

All subjects who were administered acetaminophen or placebo, including withdrawal cases, were included into the analyses. Differences in the mean, median and proportion between the acetaminophen group and the placebo group were tested by the Student's t-test or Fisher's exact test, as appropriate. $\mathrm{P}<0.05$ was considered as statistically significant for all analyses; all analyses were two-tailed. Correlations between maximum ALT level $\left(\mathrm{ALT}_{\max }\right)$ and pharmacokinetic parameters, and $\mathrm{ALT}_{\max }$ and HMGB-1 were calculated by the Pearson's correlation coefficient. All analyses were performed using JMP 11.0 (SAS Institute Inc).

\section{Results}

\section{Subject background}

The flow diagram of the subjects in this study is shown in Figure 1. Of the 435 subjects screened, 242 were allocated to either the acetaminophen group $(n=202)$ or the placebo group $(n=40)$ and 193 subjects, who did not satisfy the inclusion criteria or who met the exclusion criteria, were withdrawn from the study. Twenty subjects in the acetaminophen group were withdrawn from the study. Their reasons for withdrawal included: ALT, AST, or ALP elevation $(>2 \times U L N)(n=13)$; administration of other $\operatorname{drug}(\mathrm{s})$ that could affect this study $(\mathrm{n}=1)$, safety concerns such as serious adverse events (anaphylaxis: $n=1$ ) ; withdrawal of consent $(n=1)$; and an attending physician's judgment that study continuation would be unsuitable because of poor compliance to the study drug $(n=4)$. In addition, three subjects in the placebo group were withdrawn from the study; two subjects experienced safety concerns (headache, unusual abdominal sensation, and allergic reaction) and one subject was deemed inappropriate to continue the study by an attending physician because of poor compliance to the study drug $(n=1)$.

The background of the subjects in each group is summarized in Table 1. No significant differences were observed in male-tofemale ratio, age, BMI, history of alcohol intake, baseline ALT, or baseline AST between the acetaminophen group and the placebo group. In this subject population, both baseline AST and ALT levels were significantly higher in male subjects than in female subjects (Table 2).

\section{ALT profile during the study period}

The changes in ALT levels during the study period are shown in Figure 2 by group and gender. In both groups, ALT was higher in male subjects than in female subjects throughout the study period. ALT in the acetaminophen group increased from Day 7 to Day 14 after the start of acetaminophen administration and was higher than in the placebo group during that period. However, this ALT elevation was attenuated after Day 14. In the placebo group, a transient increase in ALT was observed on Day 7 in men, which soon returned to baseline; no particular changes in ALT were observed in women in the placebo group during the study period. The measurement day at which the subject's maximum ALT level $\left(\mathrm{ALT}_{\max }\right)$ was observed was computed for each group. $\mathrm{ALT}_{\max }$ tended to occur on Day 7 or later in the acetaminophen group, but tended to appear before Day 7 in the 


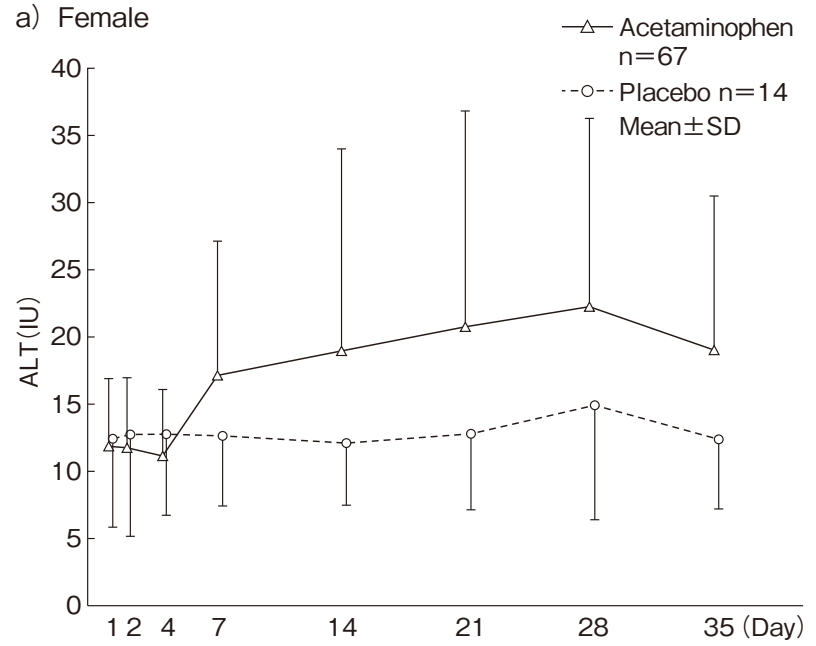

b) Male

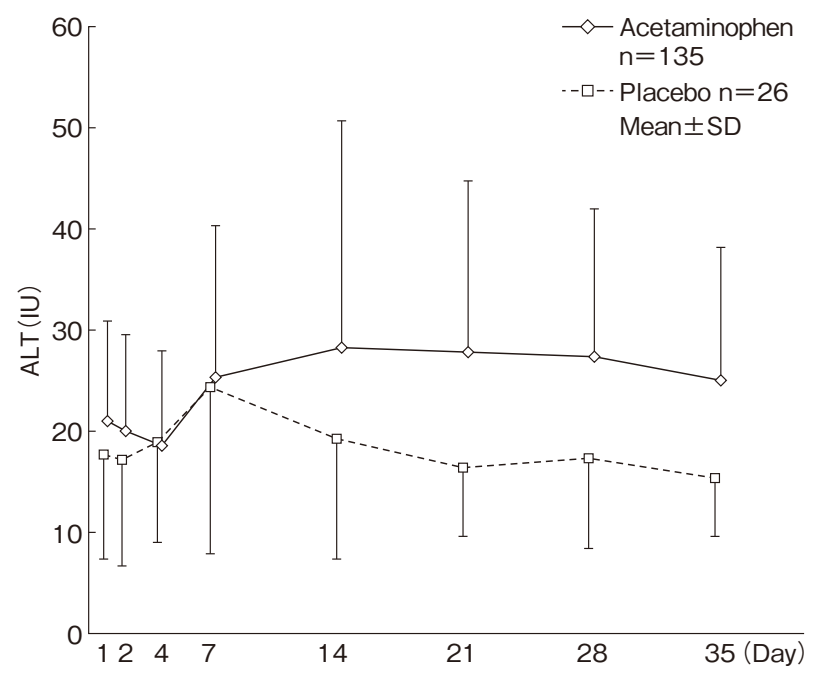

Figure 2 Time course of ALT after $3000 \mathrm{mg} /$ day of acetaminophen or placebo

placebo group (Figure 3). The frequencies of $\mathrm{ALT}_{\max }$ at $>1 \times$ $\mathrm{ULN},>2 \times \mathrm{ULN}$, and $>3 \times \mathrm{ULN}$ were also compared: $\mathrm{ALT}_{\max }$ values greater than ULN were more frequently observed in men than in women in both groups. Moreover, 12 subjects in the acetaminophen group showed $\mathrm{ALT}_{\max }$ values of $>2 \times \mathrm{ULN}$, one of the withdrawal criteria, and one subject showed $>3 \times$ ULN, one of Hy's Law criteria (ALT or AST levels of $>3 \times$ ULN and

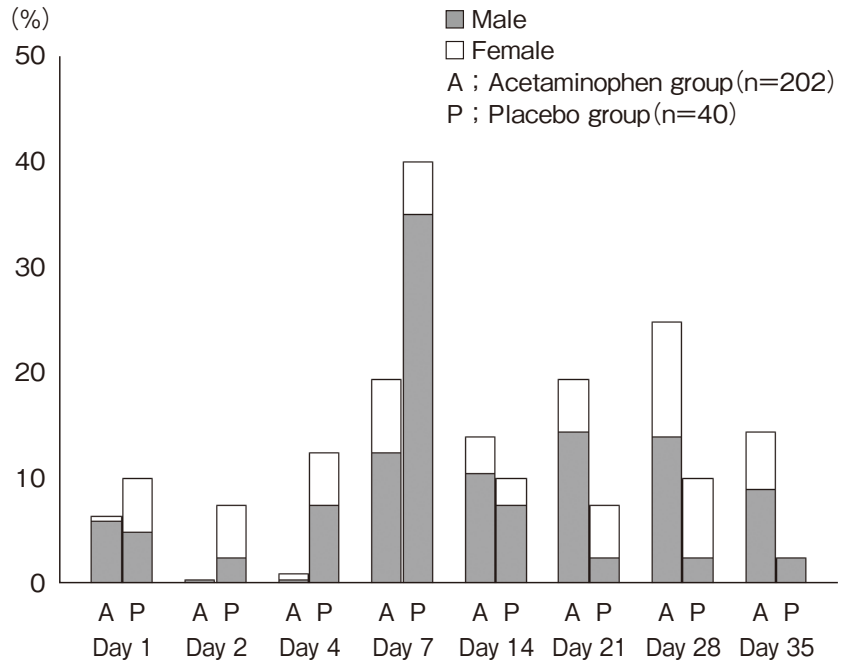

Figure 3 Histogram of the measurement day on which $\mathrm{ALT}_{\max }$ was observed

T-Bil levels of $>2 \times$ ULN) for hepatic injury; however, no subjects in the placebo group showed $>2 \times$ ULN ALT $_{\max }$ (Table 3). The findings suggested that ALT increased from Day 7 after the start of acetaminophen administration and the effect was greater in men than in women.

\section{Effects of subject background factors on ALT levels}

The association of individual factors with $\mathrm{ALT}_{\max }$ in the acetaminophen group was examined by the division of the subjects into groups based on the ULN of $\mathrm{ALT}_{\max }$ (the $>1 \times$ $\mathrm{ULN}_{\mathrm{ALT}_{\max }}$ group and the $\leq 1 \times \mathrm{ULN} \mathrm{ALT}_{\max }$ group) and the examination of the effects of gender, age, BMI, and history of alcohol intake. Significant differences in gender and age were observed between the $>1 \times \mathrm{ULN}$ and $\leq 1 \times$ ULN subgroups (Table 4). None of the pharmacokinetic parameters examined

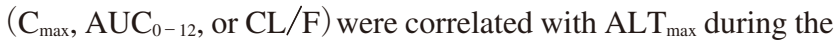
study period (Figure 4).

\section{ALT profile in subjects with ALT elevation}

For subjects who showed a clear ALT elevation $(>1 \times \mathrm{ULN}$ $\left.\mathrm{ALT}_{\text {max }}\right)$, the ALT profiles were examined for the adaptation phenomenon. Similar to the results shown in Figure 2, most subjects showed transient, rather than persistent, elevation in both groups. $\mathrm{ALT}_{\max }$ was observed on or before Day 7 in all subjects in the placebo group, whereas the peak was observed on Day 14 or later in some subjects in the acetaminophen group

Table 3 Prevalence of subjects with ALT $_{\max }$ over upper limit of normal

\begin{tabular}{|c|c|c|c|c|c|c|}
\hline \multirow[b]{2}{*}{$\mathrm{ALT}_{\max }$} & \multicolumn{3}{|c|}{ Acetaminophen } & \multicolumn{3}{|c|}{ Placebo } \\
\hline & $\begin{array}{c}\text { Total } \\
\mathrm{N}=202\end{array}$ & $\begin{array}{c}\text { Male } \\
\mathrm{N}=135\end{array}$ & $\begin{array}{l}\text { Female } \\
\mathrm{N}=67\end{array}$ & $\begin{array}{c}\text { Total } \\
\mathrm{N}=40\end{array}$ & $\begin{array}{c}\text { Male } \\
\mathrm{N}=26\end{array}$ & $\begin{array}{l}\text { Female } \\
\mathrm{N}=14\end{array}$ \\
\hline$>1 \times$ ULN, $N(\%)$ & $69(34.2)$ & $56(41.5)$ & $13(19.5)$ & $5(12.5)$ & $5(19.2)$ & $0(0)$ \\
\hline$>2 \times$ ULN, N $(\%)$ & $12(5.9)$ & $9(6.7)$ & $3(4.5)$ & $0(0)$ & $0(0)$ & $0(0)$ \\
\hline$>3 \times$ ULN, N $(\%)$ & $1(0.5)$ & $1(0.7)$ & $0(0)$ & $0(0)$ & $0(0)$ & $0(0)$ \\
\hline
\end{tabular}

ULN ; upper limit of normal 
Table 4 Subject characteristics with elevated ALT after the administration of acetaminophen

\begin{tabular}{lccc} 
& $\begin{array}{c}\mathrm{ALT}_{\max }>1 \times \mathrm{ULN} \\
\mathrm{N}=69\end{array}$ & $\begin{array}{c}\mathrm{ALT}_{\max } \leqq 1 \times \mathrm{ULN} \\
\mathrm{N}=133\end{array}$ & P value \\
\hline Male, $\mathrm{N}(\%)$ & $56(81.2)$ & $79(59.4)$ & $<0.001$ \\
Age, mean $\pm \mathrm{SD}$ & $33.4 \pm 8.6$ & $30.9 \pm 7.6$ & 0.028 \\
BMI, mean $\pm \mathrm{SD}$ & $22.2 \pm 2.1$ & $21.8 \pm 2.0$ & 0.150 \\
Self-reported alcohol use, N (\%) & $25(36.2)$ & $63(47.4)$ & 0.159 \\
\hline
\end{tabular}

Categorical data are shown as percentage of subjects. Continuous data are shown as mean and standard deviation. P value were calculated by Fisher's exact test for categorical data or t-test for continuous data.
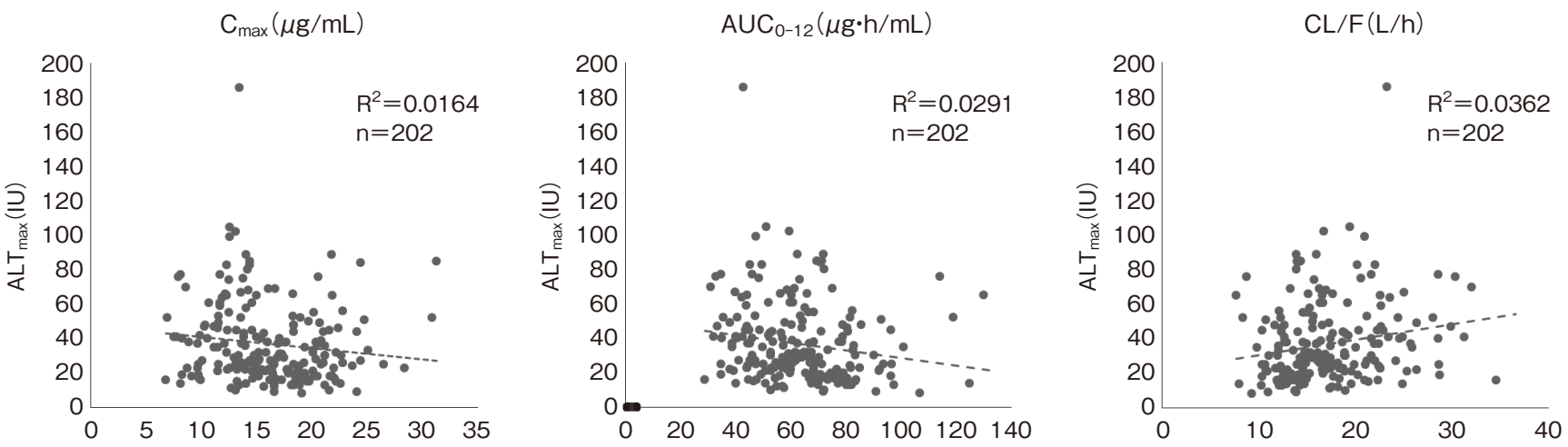

Figure 4 Correlation between pharmacokinetic parameters and $\mathrm{ALT}_{\max }$ in acetaminophen group

$\mathrm{AUC}_{0-12} ;$ area under the curve $0-12$ hour, $\mathrm{C}_{\max } ;$ maximum drug concentration, $\mathrm{CL} / \mathrm{F}$; apparent clearance $\mathrm{R}$; correlation coefficient

a) Acetaminophen

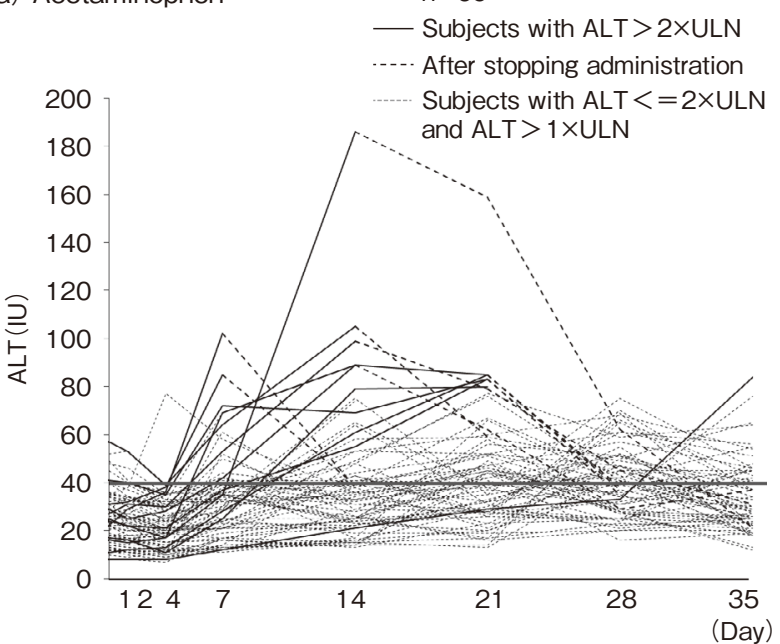

b) Placebo

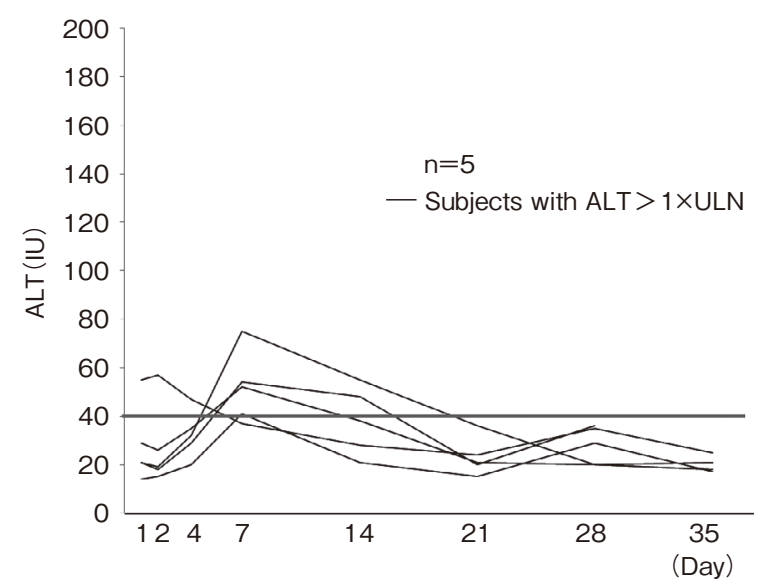

Figure 5 Individual ALT profiles of subjects with ALT $>1 \times$ ULN was observed during the study period ULN; upper limits of normal

(Figure 5). We then investigated the change in ALT (dALT), rather than the absolute ALT level, and examined the difference in ALT concentration between two consecutive measurement time points in a given individual. The correlation analysis of $\mathrm{ALT}_{\max }$ for each subject in the acetaminophen group subjects showed that higher $\mathrm{ALT}_{\max }$ values resulted in larger decreases in ALT at the next measurement point (Figure 6). For this analysis, 109 subjects whose $\mathrm{ALT}_{\max }$ and ALT at the next occasion were measured during the administration of acetaminophen were included.

\section{HMGB-1 as a biomarker for hepatic injury}

A correlation was not observed between HMGB-1 in the acetaminophen group and $\mathrm{ALT}_{\max }$ during the study period (Figure 7).

\section{Discussion}

In this study, we administrated the liver function test to healthy 


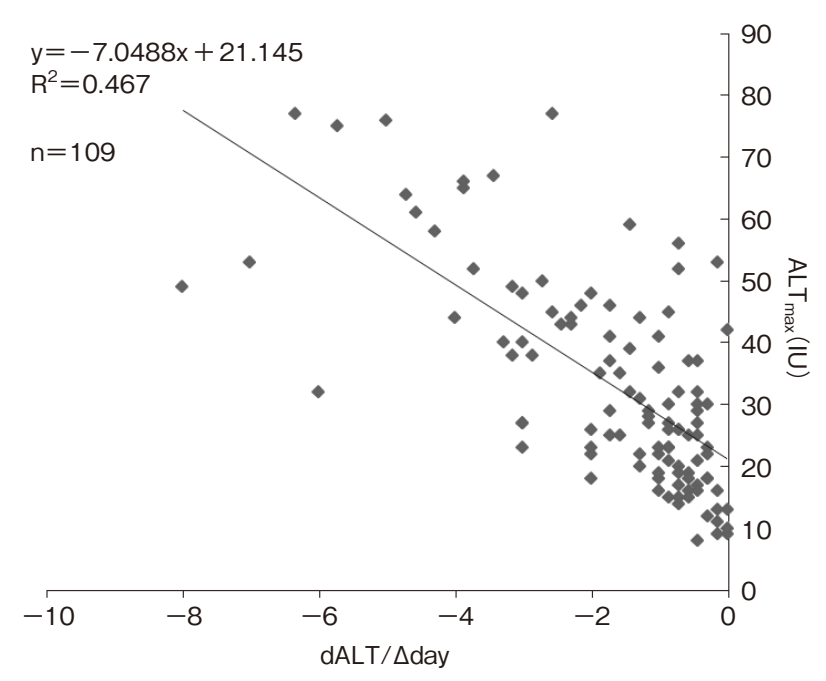

Figure 6 Correlation between $\mathrm{ALT}_{\max }$ and consecutive changes in ALT during administration acetaminophen

dALT ; difference in ALT between ALT max $_{\text {ax }}$ and ALT measured on the next occasion during administration of acetaminophen. $\triangle$ day ; number of days between the occasions 2 consecutive ALT measurements.

$\mathrm{R}$; correlation coefficient

adults for 28days with acetaminophen $(3000 \mathrm{mg} /$ day $)$. Gender differences in ALT and AST level are well established, ${ }^{9,10)}$ this study indicated that baseline ALT was dependent on gender and that high $(\geq 1 \times$ ULN $)$ ALT was associated with the background factors of gender and age. In response to long-term, high-dose acetaminophen administration, ALT elevation higher than $2 \times$ ULN was observed in $12 / 202$ subjects $(6.0 \%)$ of the acetaminophen group; however, no subjects met Hy's Law criteria for hepatic injury. Therefore, although hepatic injury should be evaluated with reference to ULN values as a general rule, professionals must be aware that the parameter may indicate many false-positive cases owing to the variation of ALT levels among individuals.

Interestingly, no correlation was observed between the various pharmacokinetic parameters examined and $\mathrm{ALT}_{\max }$. As these parameters were calculated following a single dose in this study, our results may not adequately reflect the effects of long-term acetaminophen administration. In future, analysis would ideally incorporate pharmacokinetic parameters and the effects of metabolites after repeated doses in order to accurately assess the correlation between ALT fluctuations and pharmacokinetics.

$\mathrm{ALT}_{\max }$ concentration was observed in all subjects in the placebo group within 7 days of the start of drug administration, but was observed on day 7 or later for subjects in the acetaminophen group. The ALT elevation in the placebo group may have been influenced by the changes in living environment. The ALT elevation in the placebo group was smaller than in the acetaminophen group and fewer subjects showed an $\mathrm{ALT}_{\max }$ of $>1 \times \mathrm{ULN}$; moreover, $\mathrm{ALT}_{\max }$ occurred within 7 days of the start of drug administration in all subjects in the placebo group. These findings suggested that the change in living environment to a restricted, in-patient hospital setting may have influenced the nature of the ALT elevation in the placebo group. ${ }^{11-13)}$ In

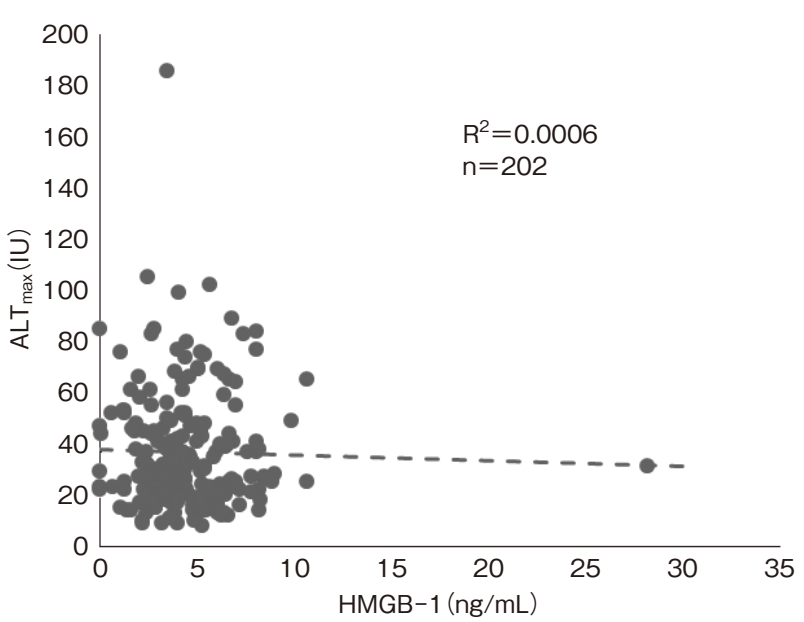

Figure 7 Correlation between $\mathrm{ALT}_{\max }$ and HMGB-1 in acetaminophen group

HMGB-1 ; High Mobility Group Box 1 (measured at the same thing with $\mathrm{ALT}_{\max }$ )

$\mathrm{R}$; correlation coefficient

contrast, in the acetaminophen-administered group, there are several subjects who showed $\mathrm{ALT}_{\max }$ on the 7th day after the start of taking investigational drugs, so there is a possibility that they may be affected by hospital restraint as well. However, when considered individual cases, 3 cases of acetaminophen group developed ALT $>2 \times$ ULN on the Day 7 and were excluded from the study while no such case was found in the placebo group. And average ALT in the acetaminophen group was elevated from Day 7 to Day 14 after the start of acetaminophen, and then it was attenuated after Day 14. This ALT behavior has also been reported after the administration of other drugs and may be attributed to a phenomenon known as "adaptation". ${ }^{14)}$ Here, adaptation means a reduction in a marker, such as ALT, to the normal level by control of the in vivo response through continued drug administration, even though the marker was temporarily increased by drug administration. This research suggests the possibility that an adaptation-like phenomenon has occurred.

The concept of adaptation led us to analyze the data in terms of changes in ALT. Higher $\mathrm{ALT}_{\max }$ values were associated with lower ALT at the next measurement. That is, although acetaminophen administration resulted in the transient elevation of ALT and other test markers, an adaptation-like phenomenon was observed, in which biological control mechanisms reduced the marker levels down to the normal range during drug administration. Although the small sample size makes a definitive statement based on the results of this study difficult, the extent of the ALT elevation that can be mitigated is limited: subjects with chronic ALT elevation beyond this threshold(i.e., with 'faulty' adaptation) are at particular risk for serious hepatic injury.

Although ALT is used in clinical practice as a marker for liver disorder, it often results in false-positives because of the effects of the abovementioned adaptation. Therefore, we focused on HMGB-1, which was recently discovered as a candidate biomarker for liver disorder. The ALT elevation observed in this study appeared not to be caused by hepatic injury, as no 
correlation of HMGB-1 and $\mathrm{ALT}_{\max }$ was observed in the acetaminophen group during the study period.

ALT is generally used as a hepatic disease marker, but it could likely be used to evaluate hepatic injury more accurately if the change over time was included in addition to the absolute concentration in analysis. In addition, since no increase in HMGB-1 was observed against the increase in ALT value in this study, it is considered that necrosis of hepatocytes did not occur. Conceivably, as an ALT elevation of $2 \times \mathrm{ULN}$ may not necessarily be caused by hepatic injury, continuous administration may be an option in clinical practice, even after the observation of mild ALT elevation.

Nonetheless, existing markers, such as ALT and AST are known to be elevated in various conditions other than hepatic injury; a hepatic injury-specific marker is considered essential. Further research should be conducted on novel markers for hepatic injury and evaluation methods that can provide more accurate assessments of liver dysfunction.

\section{Conclusion}

The value of ALT showed slight increase in subjects administered by $3000 \mathrm{mg}$ daily for 4weeks when compared with in placebo group after Day 7 to Day 14. The prevalences of ALT $>2 \times \mathrm{ULN}$ were $5.9 \%$ in acetaminophen and $0 \%$ in placebo and those of ALT $>1 \times$ ULN were $34.2 \%$ and $12.5 \%$ respectively. However, this elevation was not accompanied by elevation in HMGB-1, so it's considered that this elevation was not caused by direct liver injury. Also, the ALT value tended to decrease after the ALT value increased in many subjects, conceivably adaptation-related biological control mechanisms caused it return to normal levels even during the continued administration of the drug. It is difficult to assess hepatic injury on the basis of existing liver function markers only: thus, novel markers with increased specificity to hepatic injury are required.

\section{Conflicts of interest}

All of the authors, except the corresponding author, report they have no conflict of interest to disclose. The corresponding author (Y. K.) received honoraria for medical consultants and lectures from Ayumi Pharmaceutical CO., LTD.

\section{References}

1) Larson AM, Poison J, Fontana RJ, Davern TJ, Lalani E, Hynan LS, et al. Acetaminophen-induced acute liver failure: results of a United States multicenter, prospective study. Hepatology. 2005; 42 (6): 1364-72.

2) Jaeschke H, Xie Y, McGill MR. Acetaminophen-induced liver injury: from animal models to humans. J Clin Transl Hepatol. 2014; 2 (3) : 153-61. doi : 10.14218/JCTH.2014.00014.
3) James LP, Mayeux PR, Hinson JA. Acetaminophen-induced hepatotoxicity. Drug Metab Dispos. 2003; 31 (12) : 1499-506. doi : 10.1124/ dmd.31.12.1499.

4) Antoine DJ, Dear JW, Lewis PS, Platt V, Coyle J, Masson M, et al. Mechanistic biomarkers provide early and sensitive detection of acetaminophen-induced acute liver injury at first presentation to hospital. Hepatology. 2013; 58 (2) : 777-87. doi: 10.1002/hep. 26294.

5) Watkins PB, Kaplowitz N, Slattery JT, Colonese CR, Colucci SV, Stewart PW, et al. Aminotransferase elevations in healthy adults receiving 4 grams of acetaminophen daily: a randomized controlled trial. JAMA. 2006; 296 (1) : 87-93.

6) Heard K, Green JL, Anderson V, Bucher-Bartelson B, Dart RC. A randomized, placebo-controlled trial to determine the course of aminotransferase elevation during prolonged acetaminophen administration. BMC Pharmacol Toxicol. 2014; 15: 39. doi: 10.1186/20506511-15-39.

7) FDA. Guidance for Industry Drug-Induced Liver Injury; Premarketing Clinical Evaluation. July 2009. [https://www. fda. gov/downloads/ Drugs/.../guidances/UCM174090.pdf(accessed 2017-5-12) ]

8) Kumagai Y, Tanaka R, SONG I, Sakamoto Y. Analysis of data from special drug use surveillance on elevation of liver function tests in Japanese patients administered high dose acetaminophen. Jpn J Clin Pharmacol Ther. 2016; 47 (2) : 31-7. (in Japanese). http: //doi.org/ 10.3999/jscpt.47.31.

9) Esumi Y, Tomimura K, Fukada Y. Evaluation of changes in activity of serum enzymes (AST, ALT, $\gamma$-GT) with age and gender differences. Jap J Rural Med. 2001 ; 49 (5) : 750-7. (in Japanese). doi : org/10.2185/ $\mathrm{jjrm} / 49.750$.

10) Kobayashi M. Consideration on high frequency of slightly ascending values of serum alanine aminotransferase (ALT) measurements of males: it's concerned with synthesis of proteins related to lipid metabolism. Journal of Clinical Laboratory Medicine. 1997; 41: 71521. (in Japanese)

11) Kanamaru M, Nagashima $S$, Uematsu $T$, Nakashima M. Influence of 7day hospitalization for phase I study on the biochemical laboratory tests of healthy volunteers. Jap J Clin Pharmacol Ther. 1989; 20 (3): 493-503. (in Japanese). http: //doi.org/10.3999/jscpt.20.493.

12) Kobayashi M, Yamada N, Shibata $H$, Nishikawa $T$. Elevated serum transaminase values in volunteers after administration of placebo in a phase I study. Jap J Clin Pharmacol Ther. 1993; 24 (3) : 493-6. http: //doi.org/10.3999/jscpt.24.493.

13) Azuma J, Seto Y, Mochizuki N, Maruyama K, Ogura M. Changes in the serum enzyme levels and the predicting factors during 2 weeks hospitalization in healthy male volunteers: a retrospective study changes in serum levels of GOT, GPT, r-GTP, LDH, ALP and LAP. Jap J Clin Pharmacol Ther. 24 (2) : 385-96. http: //doi.org/10.3999/ jscpt.24.385.

14) Lewis JH, Mortensen ME, Zweig S, Fusco MJ, Medoff JR, Belder R. Efficacy and safety of high-dose pravastatin in hypercholesterolemic patients with well-compensated chronic liver disease: results of a prospective, randomized, double-blind, placebo-controlled, multicenter trial. Hepatology. 2007; 46 (5) : 1453-63. 